\title{
Protein Structure-based Design of Anti-protozoal Drugs
}

\author{
Christophe L. M. J. Verlinde ${ }^{*, a, b}$, Jerome C. Bressi ${ }^{c}$,Jungwoo Choe ${ }^{a}$, Stephen Suresh ${ }^{a}$, Frederick S. \\ Buckner ${ }^{d}$, Wesley C. Van Voorhis ${ }^{d}$, Paul A. M. Michels ${ }^{f}$, Michael H. Gelb ${ }^{b}$ and Wim G. J. Hol ${ }^{a, b, e}$ \\ ${ }^{a}$ Biomolecular Structure Center,${ }^{b}$ Departments of Biochemistry, ${ }^{c}$ Chemistry, ${ }^{d}$ Medicine, ${ }^{e}$ Howard Hughes \\ Medical Institute, University of Washington, Box 357742, Seattle, WA 98195-7742, USA \\ ${ }^{f}$ Research Unit for Tropical Diseases, Christian de Duve Institute of Cellular Pathology, Brussels, Belgium
}

\begin{abstract}
O repertório de drogas disponível para o combate de doenças causadas por protozoários, tais como a doença de Chagas, leishmaniose e doença de sono, é deploravelmente inadequado. Atualmente, os projetos de seqüenciamento genômico e genoma estrutural elucidam rapidamente novos alvos terapêuticos, proporcionando com isso oportunidades extraordinárias para os químicos medicinais. Aqui nós ilustramos a utilidade do "desenho de drogas baseado na estrutura" nesse processo, utilizando como exemplo nossos esforços para bloquear seletivamente a glicólise em tripanosomatideos.
\end{abstract}

The repertory of drugs to fight protozoal diseases such as malaria, Chagas' disease, leishmaniasis, and African trypanosomiasis is woefully inadequate. Now, genome sequencing and structural genomics projects are quickly elucidating new drug targets, providing incredible opportunities for medicinal chemists. Here, we illustrate the power of structure-based drug design in this process by our efforts to selectively block trypanosomal glycolysis.

Keywords: drug design, Chagas' disease, leishmaniasis, malaria, African trypanosomiasis, GAPDH

\section{Introduction}

\section{Tropical protozoal diseases: a challenge}

The World Health Report 2000 lists 55 million deaths in 1999 due to all causes. Tragically, specific protozoal diseases occupy a prominent place in this list. 1,086,000 people died from malaria, 66,000 from African trypanosomiasis, 21,000 from Chagas' disease, 14,000 from schistosomiasis, and 57,000 from leishmaniasis. ${ }^{1}$ Premature death captures only part of the picture. These sobering statistics are further supplemented with burden of disease data expressed in DALYs, disability-adjusted life years robbed by disease: 45 million by malaria, 2 million by trypanosomiasis; 0.7 million by Chagas' disease; 2 million by schistosomiasis; and 2 million by leishmaniasis. ${ }^{1}$ In turn, a large burden of disease impedes economical growth and prosperity, further compounding the misery. ${ }^{2}$

At first sight, little change in this sad situation is to be

*e-mail: verlinde@u.washington.edu expected. The geographical distribution of these diseases is mainly centered about the tropics, where the climate favors persistence of the vectors for transmission. Vector control is possible, eradication probably not. ${ }^{3}$ Vaccine development has not been successful thus far, mainly because parasites are experts at evading or dysregulating the human immune system. For example, trypanosomes can switch among 1000 genes encoding for their surface glycoprotein, and malaria-infected erythrocytes adhere to the antigen-presenting dendritic cells, thereby preventing their maturation and the proper mounting of an immune response. ${ }^{4,5}$ Chemotherapy to fight the mentioned diseases exists, but is plagued by high toxicity and increasing resistance. For example, melarsoprol, the only drug that can cure late-stage T.brucei rhodesiense trypanosomiasis, kills $5 \%$ of the patients. ${ }^{6}$ Adding insult to injury, pharmaceutical companies steer away from vaccine and drug development to fight tropical diseases because such programs do not fit in their profit-driven business model. Yet, many new opportunities are on the horizon thanks to fundamental parasitology research, parasite genome sequencing efforts, advances in structural biology methods, 
the ability to generate many molecules by combinatorial chemistry, and the integration of the latter into structurebased drug design methods.

\section{New opportunities for chemotherapy}

The current list of validated targets for new drugs against trypanosomatids includes glycolytic enzymes, trypanothione reductase, cathepsin L-like proteases, various kinases, and protein farnesyl tranferase. ${ }^{7}$ New targets in Plasmodium are protein prenylation, hemoglobin degradation in the food vacuole, mitochondrion electron transport, and apicoplast metabolism. ${ }^{8}$ The discovery of the apicoplast, a chloroplast-like organelle, was completely unexpected and due to genomics. Yet, more surprises are to be expected. The malaria genome sequencing effort is expected to be completed by mid 2002. ${ }^{9}$ Meanwhile, the sequencing of the genomes from Trypanosoma brucei, Trypanosoma cruzi, Schistosoma mansoni, and Leishmania major are underway. ${ }^{10}$ Also, 2001 saw the birth of an academic consortium, named SGPP (= Structural Genomics for Pathogenic Protozoa; see: www.sgpp.org). This consortium plans to determine the three-dimensional structures of several hundreds of proteins from T.brucei, T. cruzi, various Leishmania sub-species, and Plasmodium falciparum. Hence, medicinal chemists will have a quickly expanding list of opportunities to design and synthesize the anti-protozoal drugs of the future.

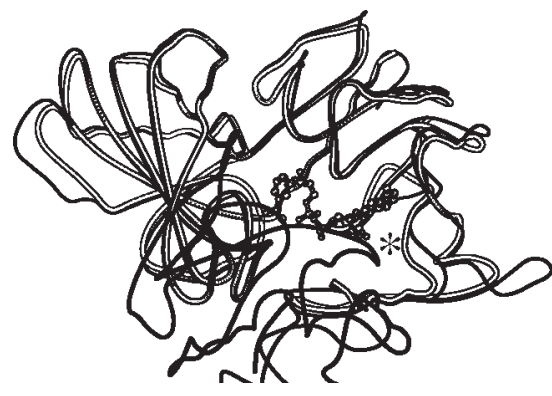

Figure 1. Superpositon of T. brucei (black line) and human GAPDH (open line). For clarity, only one subunit and part of a neighboring of the tetramers are shown. An asterisk denotes the divergence of the protein backbones near the adenosine of the NAD co-factor.

\section{Results and Discussion}

\section{Trypanosomal glycolysis: a case study}

To illustrate the power of structure-based drug design we will discuss here our efforts at targeting trypanosomal glycolysis. In the human host, African trypanosomes live in the bloodstream. There, they derive all their energy from glycolysis to the stage of pyruvate. Most of the glycolytic enzymes are sequestered in a specialized peroxisome-like organelle, called the glycosome. ${ }^{11}$ In 1976 it was demonstrated that bloodstream form trypanosomes die within minutes when their glycolysis is blocked in vitro. ${ }^{12}$ However, to achieve the same effect without harming a patient, selective inhibitors of trypanosomal glycolytic enzymes need to be developed.

At first sight, each of the glycolytic enzymes might be a target for drug design provided that sufficient exploitable differences exists with their human counterparts. Yet, glycolytic flux control considerations also play a role in picking a target. Recent computer simulations that include the experimental kinetic constants of all T.brucei enzymes have shown that blocking aldolase glyceraldehyde-3phosphate dehydrogenase (GAPDH), glycerol-3phosphate dehydrogenase and phosphoglycerate kinase have the largest effect on the flux through the glycolytic pathway, and thus on the energy production. ${ }^{13}$ Here, we show how we targeted GAPDH.

\section{Glyceraldehyde-3-phosphate dehydrogenase: selective} inhibition

We solved the crystal structure of T.brucei GAPDH and improved the resolution of its human counterpart. ${ }^{14}$ Their active sites appeared to be identical, with the closest difference nearly $12 \AA$ away from the catalytic cysteine, dimming the hopes for selective competitive inhibitors with the substrate or product. Fortunately, the adenosine pocket for the NAD co-factor showed dramatic differences, opening perspectives for selective co-factor analog inhibitors.

Near the adenosine ribose the protein backbones diverge between the two enzymes (Figure 2). As a result the T.brucei GAPDH exhibits a hydrophobic cleft near the O2' hydroxyl. Such a cleft is absent in the human enzyme. We exploited this difference by inventing adenosine derivatives that would occupy the cleft, maintain a hydrogen bond with the carboxylate of Asp 37, and make hydrophobic interactions. Molecular mechanics calculations showed that 2'-benzamido derivatives satisfied these criteria. After synthesis it appeared that 2 'deoxy-2'-(3-methoxybenzamido)adenosine inhibited $T$. brucei GAPDH 45 times better than adenosine. ${ }^{15}$ More importantly, no evidence of human GAPDH blocking could be detected. Thus, we succeeded in obtaining a selective inhibitor.

Having solved the selectivity problem, we then focused on improving the affinity of our lead because adenosine 
a

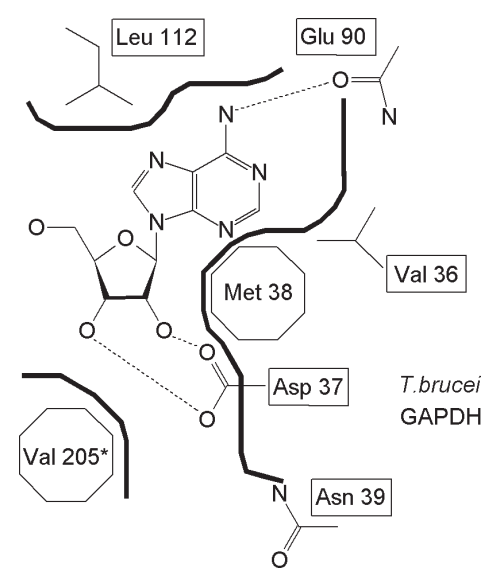

b

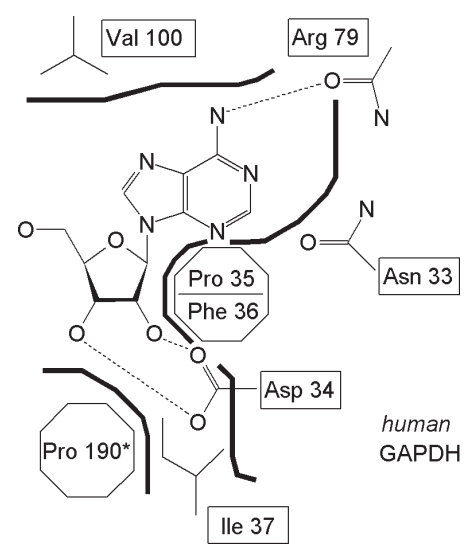

Figure 2. Schematic diagrams of the adenosine binding pockets in T.brucei (a) and human GAPDH (b).

itself is an extremely poor inhibitor of T.brucei GAPDH, with a $\mathrm{Ki}$ of $50 \mathrm{mmol} \mathrm{L}^{-1}$. Modeling showed that the introduction of a 2-thienyl at position 8 of the purine ring would bury a major part of the Leu 112 side chain. After synthesis we discovered that a 180 -fold gain in affinity with respect to adenosine was obtained this way. Unfortunately, 8-adenine substituents appeared to be sterically incompatible with 2'-(3-methoxybenzamido) because they would clash with the amido oxygen in the enzyme-fitting conformation (Figure 3). Experimental evidence for this conclusion came after we subsequently synthesized 2'-deoxy-2'-(3-methoxybenzamido)-8-(2thienyl)adenosine. This compound was only a millimolar inhibitor, worse than its mono-substituted parent compounds.

At this stage in the project we experienced problems with obtaining sufficient amounts of T.brucei GAPDH for a full-fledged drug design project. T. brucei GAPDH can only be obtained by a laborious procedure that involves infecting rats; overexpression attempts were unsuccessful. Because GAPDH of Leishmania mexicana, a parasite

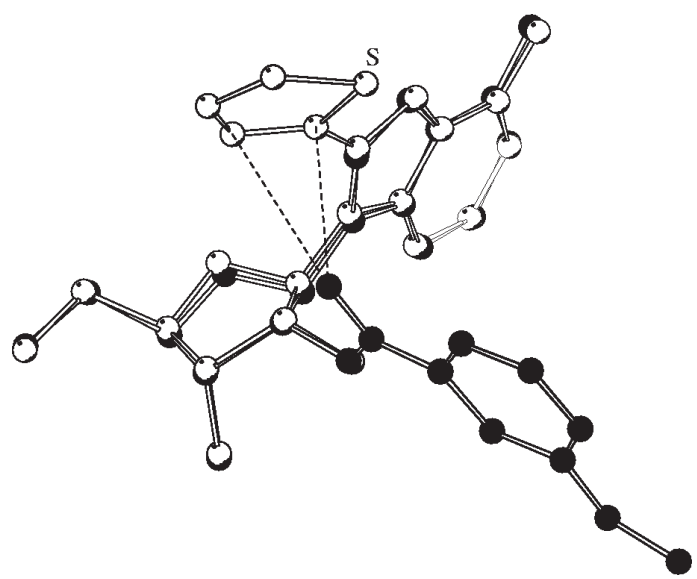

Figure 3. Superposition of 2'-deoxy-2'-(3-methoxybenzamido) adenosine (black atoms) and 8-(2-thienyl)adenosine (white atoms).

related to $T$. brucei, did not exhibit this problem we used it as a substitute in many stages of our studies. This proved to be a good strategy as our subsequent structure determination of the L. mexicana enzyme showed that their adenosine pockets are virtually identical, with a $0.2 \AA \mathrm{rms}$ deviation on backbone and $0.5 \AA$ on side chain atom positions; the only amino acid difference is the replacement of Asn 39 of T.brucei GAPDH by a Ser in L.mexicana. ${ }^{16} \mathrm{As}$ expected, L.mexicana GAPDH inhibition by our new inhibitors was nearly identical to the T.brucei results.

Subsequently, we shifted our attention to the N6 position of the purine for affinity improvement. This atom is adjacent to two hydrophobic areas on the protein surface, one formed by the side chains of Leu 112, Phe 113 and Arg 91, another one by Met 38 and Arg 91 (Figure 4). We purchased five $\mathrm{N6}$-adenosine derivatives with hydrophobic substituents for screening, and synthesized nine others. Incorporating N6 into an amide function appeared to be detrimental, but several amines worked fine. Especially N6-benzyl-adenosine looked promising with a 10 -fold affinity gain over adenosine. ${ }^{17}$

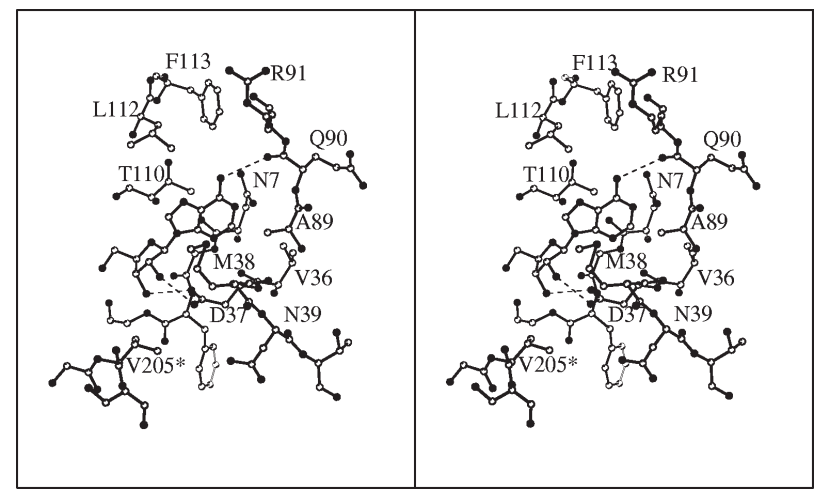

Figure 4. Stereoview of the adenosine binding pockets in $T$. brucei GAPDH. 
The benzyl substituent of N6-benzyl-adenosine could be modeled into the binding site in two different orientations. We resolved this dilemma by a crystal structure determination. Because co-crystals of parasite GAPDH with N6-benzyl-adenosine failed to grow, we synthesized the N6-benzyl-NAD analogue. With this molecule we grew co-crystals with $L$. mexicana GAPDH. The N6-benzyl appeared to be sandwiched between the side chains of Met 39 and Arg 92, corresponding to Met 38 and Arg 91 in T.brucei (Figure 5), settling the orientation dilemma. $^{18}$

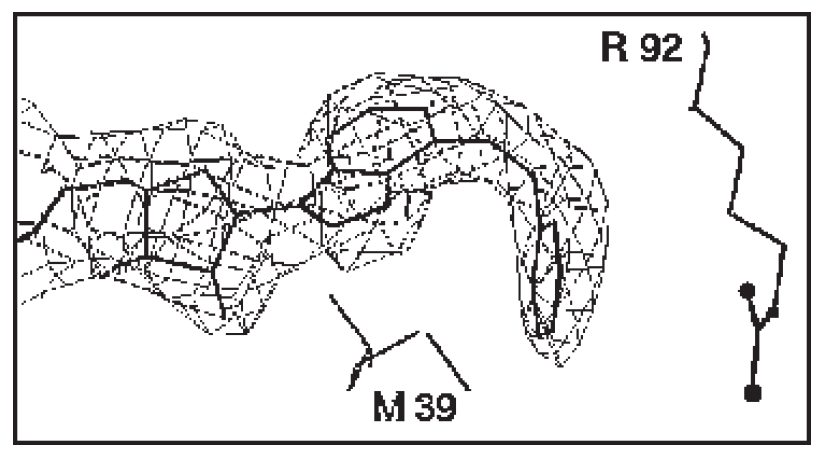

Figure 5. Experimental binding mode of N6-benzyl-NAD to L.mexicana GAPDH.

N6-benzyl-adenosine formed the basis for further affinity improvement by design. A search in the Available Chemicals Database 95.2 revealed the commercial availability of 1,124 benzylamines of which 88 appeared suitable for reaction with 6-chloropurine riboside. All 88 were modeled as N6-adenosine derivatives in the $T$. brucei binding site. Poorly fitting molecules were rejected. After similarity clustering we decided on the synthesis of six of them. All six proved to be more potent than N6-benzyladenosine (Table 1). The best compound was N6-(1naphtalenemethyl)adenosine, with a 333-fold gain in affinity over adenosine. ${ }^{17}$

Several of the N6-substituents were combined with the 2'-(3-methoxybenzamido) substituent, leading to potent

Table 1. Inhibition of L.mexicana GAPDH by N6-adenosine derivatives

\begin{tabular}{lc}
\hline N6-substituent & IC50 $\left(\mu \mathrm{mol} \mathrm{L}^{-1}\right)$ \\
\hline benzyl & 4,200 \\
2-methylbenzyl & 700 \\
3-methylbenzyl & 750 \\
1,2,3,4-tetrahydro-1-naphtyl & 360 \\
1-naphthalenemethyl & 150 \\
2-[2-(hydroxymethyl)phenylthio]-benzyl & 340 \\
diphenylmethyl & 240 \\
\hline
\end{tabular}

and selective inhibition (Table 2). In particular the 1naphthalenemethyl derivative afforded submicromolar inhibition against L.mexicana GAPDH. ${ }^{18}$ Its modeled binding mode is shown in Figure 6. The new compounds were slightly less potent against the T.brucei and T.cruzi enzymes, which share the same amino acids in the targeted area. None of the designed compounds inhibited the human GAPDH to any degree at submillimolar concentrations, their upper solubility limit.

Table 2. Selective parasite GAPDH inhibition by N6-substituted 2'deoxy-2'-(3-methoxybenzamido)adenosine derivatives (IC50 in $\mu \mathrm{mol} \mathrm{\textrm {L } ^ { - 1 } )}$

\begin{tabular}{lllll}
\hline N6-substituent & L. mexicana & T. brucei & T.cruzi & Human \\
\hline benzyl & 16 & 159 & 160 & $>530^{\mathrm{a}}$ \\
2-methylbenzyl & 4 & 40 & 35 & $>270^{\mathrm{a}}$ \\
1-naphthalenemethyl & 0.2 & 2 & 5 & $>200^{\mathrm{a}}$ \\
\hline
\end{tabular}

a insoluble above and non-inhibitory at stated concentration

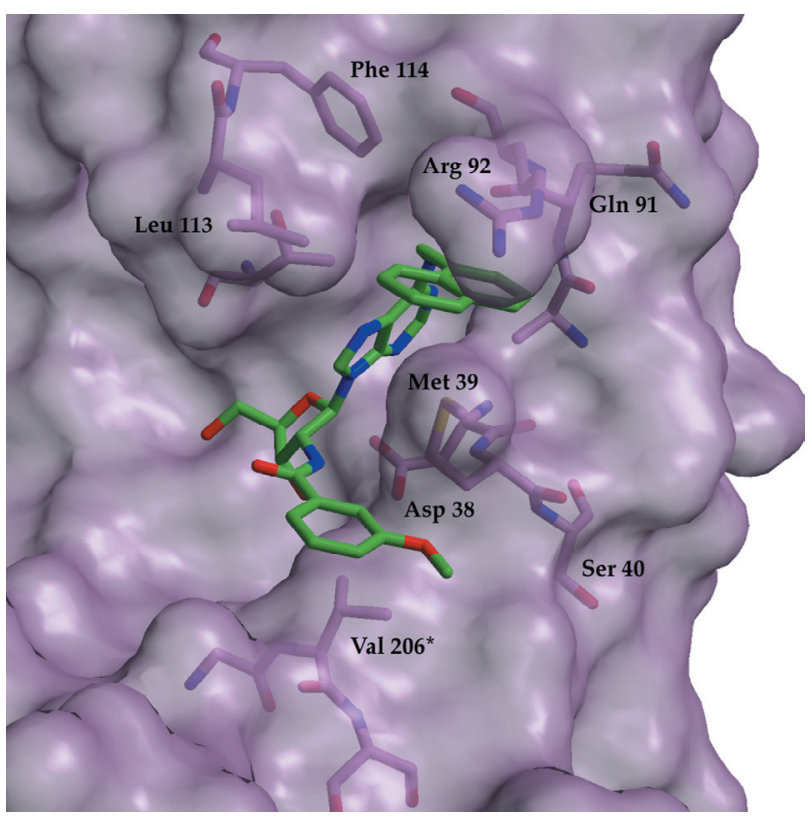

Figure 6. Predicted binding mode of 2'-deoxy-2'-(3-methoxybenzamido)-N6-(1-naphthalenemethyl)adenosine to L. mexicana GAPDH.

When tested against cultured bloodstream T. brucei, N6-( 1 - naphthalene me thy 1)-2' - (3-ch 1 or obenzamido)adenosine inhibited growth in the low micromolar range. ${ }^{18}$ Within minutes after adding this compound to bloodstream T. brucei, production of glucose-derived pyruvate ceased, parasite motility was lost, and a mixture of grossly deformed and lysed parasites was observed. Similar results were obtained with T.cruzi amastigotes (Figure 7). 


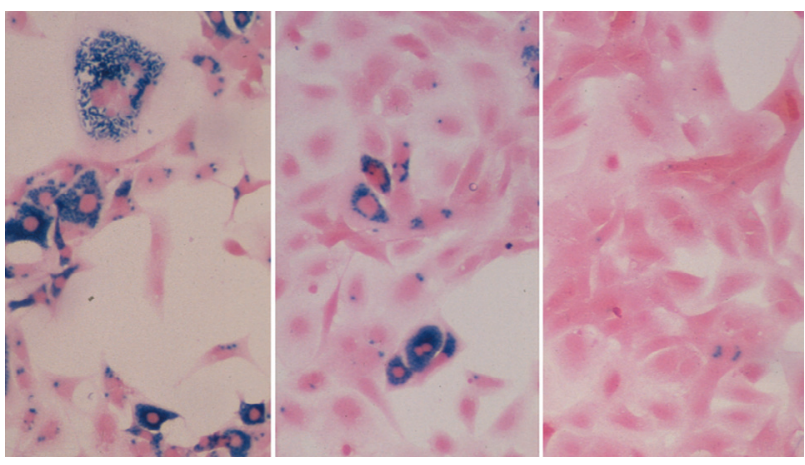

Figure 7. Optical microscopy showing $3 \mathrm{~T} 3$ fibroblasts infected with T. cruzi amastigotes on addition of 0 (Left), 16 (Middle), and $40 \mu \mathrm{mol} \mathrm{L}^{-1}$ (Right) 2'-deoxy-2'-(3-chlorobenzamido)-N6-(1-aphthalenemethyl) adenosine. After fixation, cells were stained with 5-bromo-4-chloro3 -indolyl $\beta$-D-galactoside to assay $\beta$-galactosidase and counterstained with Safranin O.

\section{Conclusion}

The present study underlines the value of a structurebased approach to dramatically improve the affinity of a 50 millimolar lead. Such leads are generally ignored by the pharmaceutical industry, which insists on micromolar leads. The fact that we obtained five orders of magnitude affinity gain in one round of drug design shows that more optimism may be warranted. Also, the current results demonstrate that selectivity can be built into inhibitors.

During the course of this project many colleagues have expressed concerns of using adenosine as a scaffold because of nature's ubiquitous use of this moiety in cofactors and co-substrates, such as NAD, FAD, ATP, etc. However, our $200 \mathrm{nM}$ inhibitor's shape is a very significant departure from that of unmodified adenosine. In fact, it may be considered structurally as different from adenosine as an HIV-protease inhibitor from angiotesinogen. Furthermore, we have tested our best inhibitor against several ATP- and NAD-dependent enzymes, such as phosphoglycerate kinase, lactate dehydrogenase and glycerol-3-phosphate dehydrogenase, and found no inhibition.

Besides GAPDH we are currently also targeting several other trypanosomatid proteins for which we determined the structure: triosephosphate isomerase, phosphoglycerate kinase, fructose-1,6-bisphosphate aldolase, glycerol-3phosphate dehydrogenase, and PEX5 (a glycosomal import protein). ${ }^{19-23}$ Recently, we also solved the structure of peptide deformylase from $P$. falciparum. ${ }^{24}$ Thanks to the SGPP initiative we launched, this list of targets will grow quickly and provide a golden opportunity for drug designers and medicinal chemists to help fight the battle against protozoal diseases.

\section{Acknowledgements}

The work on trypanosomatid glycolysis was supported by Grant AI44119 from the National Institutes of Health.

\section{Bibliography}

1. WHO; The World Health Report 2000. Health Systems: Improving Performance, World Health Organization: Geneva, 2000 .

2. Diamond, J.M.; Guns, Germs, and Steel: The Fates of Human Societies, W.W. Norton: New York, 1997.

3. Rogers, D.J.; Randolph, S.E.; Nature 1991, 351, 739; Kaneko, A.; Taleo, G.; Kalkoa, M.; Yamar, S.; Kobayakawa, T.; Bjorkman, A.; Lancet 2000, 356, 1560.

4. Borst, P.; Ulbert, S.; Mol. Biochem. Parasitol. 2001, 114, 17.

5. Urban, B.C.; Ferguson, D.J.; Pain, A.; Willcox, N.; Plebanski, M.; Austyn, J.M.; Roberts, D.J.; Nature 1999, 400, 73.

6. Pepin, J.; Milord, F.; Adv. Parasitol. 1994, 33, 1.

7. Barrett, M.P; Mottram, J.C.; Coombs, G.H.; Trends Microbiol. 1999, 7, 82; Yokoyama, K.; Trobridge, P.; Buckner, F.S.; Van Voorhis, W.C.; Stuart, K.D.; Gelb, M.H.; J. Biol. Chem. 1998, 273, 26497.

8. Ridley, R.G.; Nature 2002, 415, 686; Ohkanda. J.; Lockman, J.W.; Yokoyama, K.; Gelb, M.H.; Croft, S.L.; Kendrick, H.; Harrell, M.I.; Feagin, J.E.; Blaskovich, M.A.; Sebti, S.M.; Hamilton, A.D.; Bioorg. Med. Chem. Lett. 2001, 11, 761.

9. Hoffman S.L.; Subramanian, G.M.; Collins, F.H.; Venter, J.C.; Nature 2002, 415, 702.

10. Degrave, W.M.; Melville, S.; Ivens, A.; Aslett, M.; Int. J. Parasitol. 2001, 31, 532.

11. Opperdoes, F.R.; Borst, P.; FEBS Lett. 1977, 80, 23.

12. Clarkson, A.B.; Brohn, F.H.; Science 1976, 194, 204.

13. Bakker, B.M; Westerhoff, H.V.; Opperdoes, F.R.; Michels, P.A.M.; Mol. Biochem. Parasitol. 2000, 1.

14. Vellieux, F.M.D.; Hajdu, J.; Verlinde, C.L.M.J.; Groendijk, H.; Read, R.J.; Greenhough, T.J.; Campbell, J.W.; Kalk, K.H.; Littlechild, J.A.; Watson, H.C.; Hol, W.G.J.; Proc. Natl. Acad. Sci. USA 1993, 90, 2355.

15. Verlinde, C.L.M.J.; Callens, M.; Van Calenbergh, S.; Van Aerschot, A.; Herdewijn, P.; Hannaert, V.; Michels, P.A.M.; Opperdoes, F.R.; Hol, W.G.J.; J. Med. Chem. 1994, 37, 3605.

16. Kim, H.; Feil, I.K.; Verlinde, C.L.M.J.; Petra, P.H.; Hol, W.G.J.; Biochemistry 1995, 34, 14975.

17. Aronov, A.M.; Verlinde, C.L.M.J.; Hol, W.G.J.; Gelb, M.H.; J. Med. Chem. 1998, 41, 4790.

18. Aronov, A.M.; Suresh, S.; Buckner, F.S.; Van Voorhis, W.C.; Verlinde, C.L.M.J.; Hol, W.G.J.; Gelb, M. H.; Proc. Natl. Acad. Sci. USA 1999, 96, 4273.

19. Wierenga, R.K.; Kalk, K.H.; Hol, W.G.J.; J. Mol. Biol. 1987, $198,109$. 
20. Bernstein, B.E.; Williams, D.M.; Bressi, J.C.; Kuhn, P.; Gelb, M.H.; Blackburn, G.M.; Hol, W.G.J.; J. Mol. Biol. 1998, 279, 1137; Bressi, J.C.; Choe, J.; Hough, M.T.; Buckner, F.S.; Van Voorhis, W.C.; Verlinde, C.L.M.J.; Hol, W.G.J.; Gelb, M.H.; J. Med. Chem. 2000, 43, 4135.

21. Chudzik, D.M., Michels, P.A.M., De Walque, S., Hol, W.G.J.; J.Mol.Biol. 2000, 300, 697.

22. Suresh, S.; Turley, S.; Opperdoes, F.R.; Michels, P.A.M.; Hol, W.G.J.; Structure 2000, 8, 541.
23. Kumar, A.; Roach, C.; Hirsh, I.S.; Turley, S.; deWalque, S.; Michels, P.A.M.; Hol, W.G.J.; J. Mol. Biol. 2001, 307, 271.

24. Kumar, A.; Nguyen, K. T.; Svrivathsan, S.; Ornstein, B.; Turley, S.; Hirsh, I.; Pei, D.; Hol, W.G.J.; Structure 2002, 10, 357.

Received: April 11, 2002 Published on the web: November 8, 2002 